ISSN 2072-6651

www.mdpi.com/journal/toxins

Article

\title{
A Magnetic Nanoparticle Based Enzyme-Linked Immunosorbent Assay for Sensitive Quantification of Zearalenone in Cereal and Feed Samples
}

\author{
Xian Zhang ${ }^{1}$, Xin Wang ${ }^{1}$, Mengjiao Sun ${ }^{1}$, Xiaofeng Zhang ${ }^{2}$, Houhui Song ${ }^{3}$, Yaxian Yan ${ }^{4}$, \\ Jianhe Sun ${ }^{4}$, Xiaoliang $\mathrm{Li}^{1}$ and Weihuan Fang ${ }^{1, *}$
}

1 Institute of Preventive Veterinary Medicine and Zhejiang Provincial Key Laboratory of Preventive Veterinary Medicine, Zhejiang University, 388 Yuhangtang Road, Hangzhou 310058, China; E-Mails: zhangian073@163.com (X.Z.); wangxin20095017@126.com (X.W.); sunmengjiao265@126.com (M.S.); xlli@zju.edu.cn (X.L.)

2 Food Safety Key Laboratory of Zhejiang Province, Zhejiang Entry-Exit Inspection and Quarantine Bureau, 126 Fuchun Road, Hangzhou 310012, China; E-Mail: zxf@ziq.gov.cn

3 College of Animal Science and Technology, Zhejiang A\&F University, 88 Huanbei Road, Lin'an 311300, China; E-Mail: songhh@ zafu.edu.cn

4 School of Agriculture and Biology, Shanghai Jiaotong University, Shanghai 200240, China; E-Mails: yanyaxian@sjtu.edu.cn (Y.Y.); sunjhe@sjtu.edu.cn (J.S.)

* Author to whom correspondence should be addressed; E-Mail: whfang@zju.edu.cn; Tel./Fax: +86-571-8898-2242.

Academic Editors: Michelangelo Pascale and Maria C. DeRosa

Received: 11 September 2015 / Accepted: 13 October 2015 / Published: 20 October 2015

\begin{abstract}
A novel enzyme-linked immunosorbent assay based on magnetic nanoparticles and biotin/streptavidin-HRP (MNP-bsELISA) was developed for rapid and sensitive detection of zearalenone (ZEN). The detection signal was enhanced and the sensitivity of the assay was improved by combined use of antibody-conjugated magnetic nanoparticles and biotin-streptavidin system. Under the optimized conditions, the regression equation for quantification of ZEN was $y=-0.4287 x+0.3132\left(R^{2}=0.9904\right)$. The working range was $0.07-2.41 \mathrm{ng} / \mathrm{mL}$. The detection limit was $0.04 \mathrm{ng} / \mathrm{mL}$ and $\mathrm{IC}_{50}$ was $0.37 \mathrm{ng} / \mathrm{mL}$. The recovery rates of intra-assay and inter-assay ranged from $92.8 \%-111.9 \%$ and $91.7 \%-114.5 \%$, respectively, in spiked corn samples. Coefficients of variation were less than $10 \%$ in both cases. Parallel analysis of cereal and feed samples showed good correlation between MNP-bsELISA and liquid chromatograph-tandem mass spectrometry $\left(R^{2}=0.9283\right)$.
\end{abstract}


We conclude that this method is suitable for rapid detection of zearalenone in cereal and feed samples in relevant laboratories.

Keywords: zearalenone; immunoassay; magnetic nanoparticles; biotin-streptavidin; quantification

\section{Introduction}

Zearalenone (ZEN), a mycotoxin, is produced by Fusarium species growing on grains, mainly corn and hay exposed to high moisture during storage, such as Fusarium graminearum and Fusarium roseum [1,2]. Over time, the toxicity of ZEN in food and animal feed has been widely recognized. ZEN can cause hyperestrogenism in livestock, leading to diseases and harmful effects associated with the reproductive system such as abortion and stillbirth [3-5]. Products contaminated by ZEN compromise food safety and threaten human health. Babies and children are more vulnerable to the effects of ZEN than adults [6]. Therefore, the Joint Food and Agriculture Organization (FAO)/World Health Organization (WHO) Expert Committee on Food Additives (JECFA) has established the provisional maximum tolerable daily intake levels of $0.5 \mu \mathrm{g} / \mathrm{kg}$ of body weight per day for ZEN [7].

The detection methods of mycotoxins mainly consist of chromatography and immunology-based analytical approaches. Chromatographic methods, including thin-layer chromatography [8], liquid chromatography-tandem mass spectrometry (LC-MS/MS) [9,10], and high-performance liquid chromatography [11,12], are sensitive and produce reliable results. However, the complex preparation steps, expensive equipment, and time-consuming procedures make such methods unsuitable for routine work in many laboratories and other locations, such as farms or factories. Compared with chromatographic methods, a number of immunoassays have been developed for mycotoxins detection in agricultural products with the advantages of rapid and cost effective, such as enzyme-linked immunosorbent assay (ELISA) [13-15], colloidal gold lateral flow immunoassay [16-18], electrochemical immunosensor assay (EIA) $[19,20]$ and fluorescent linked immunosorbant assays (FLISAs) [21-23]. In case of mycotoxins, sensitive, accurate and rapid analytical methods are still needed, thus requiring new strategies for signal enhancement and time-saving procedures.

Magnetic nanoparticles are recently used in assays of biomedical and food-safety fields with the advantages of uniform diameters and even distribution in solution [24-26]. Complexes between the nanoparticles and antibodies are formed by covalent immobilization. The immobilized particles can bind with the target antigens in solution and are rapidly separated by a magnetic field [27-29]. This technology has the advantages of liquid-phase immunological reactions, reduced detection time and improved sensitivity [30]. Biotin-streptavidin coupling is one of the best characterized systems for signal amplification [31-33].

Here, we report a novel ELISA strategy (MNP-bsELISA)) for sensitive detection of ZEN in cereal and feed samples. A schematic diagram of the MNP-bsELISA is shown in Figure 1. In this assay, monoclonal antibody coated magnetic nanoparticles (MNP-Anti-ZEN) and biotinylated ZEN-BSA conjugate (ZEN-BSA-Biotin) were used and the detected format is based on the indirect competitive enzyme-linked immunosorbent assay (ic-ELISA). The test is completed in a 96-well plate by using a 
base with circular magnet. This assay is suitable for high throughput detection and proves to be more sensitive and less time-consuming than the same antibody based conventional ELISA in an earlier report [34].

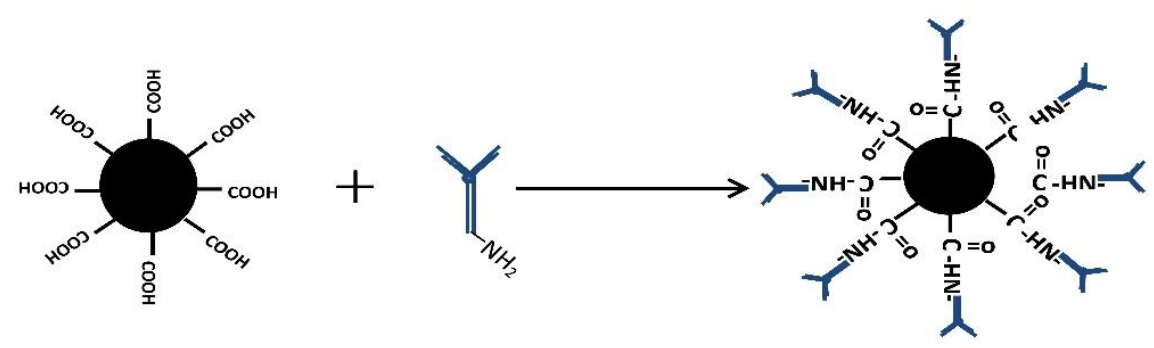

(A)
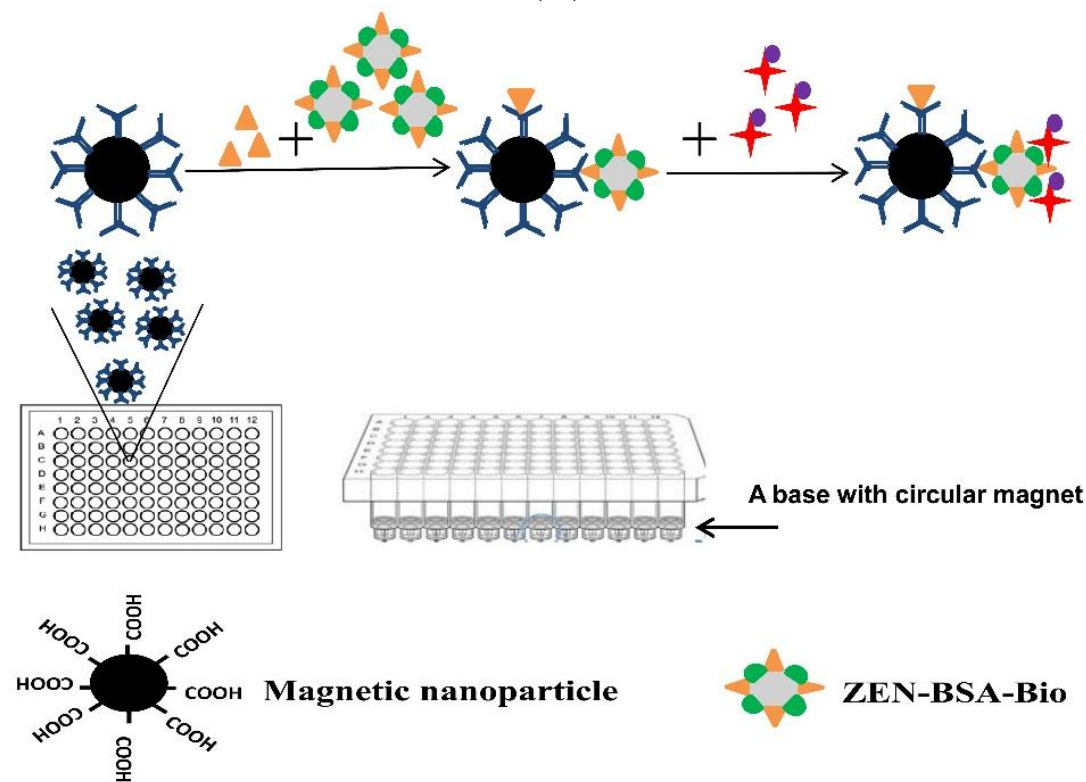

ZEN-BSA-Bio
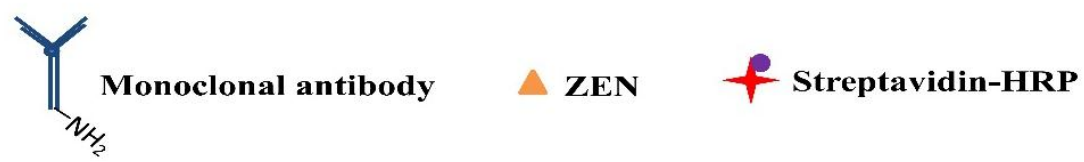

(B)

Figure 1. Schematic diagrams of the preparation of the immunomagnetic nanoparticles cross-linked with anti-zearalenone monoclonal antibody (MNP-anti-ZEN) (A) and MNP-bsELISA (B).

\section{Results and Discussion}

\subsection{Identification of ZEN-BSA Conjugate and ZEN-BSA-Biotin}

Indirect ELISA and Western blotting indicated that ZEN was successfully conjugated to the carrier protein BSA (Figure 2). There was no signal from the BSA control. For quantitation of the extent of biotin incorporation into ZEN-BSA, the dye HABA was used for colorimetric analysis of the colored complex with avidin displaced by biotin. We obtained a biotinylation level of 3.7:1 (ZEN-BSA: Biotin). 
A

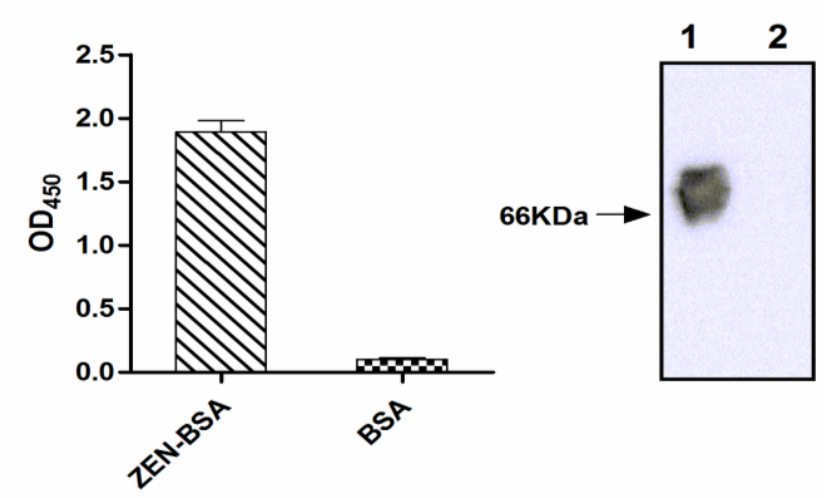

Figure 2. Identification of conjugation of zearalenone with bovine serum albumin (ZEN-BSA) by indirect ELISA (A) and Western blotting (B). The molecular weight of BSA is about $66 \mathrm{KDa}$. Lane 1: ZEN-BSA, Lane 2: BSA.

\subsection{Identification of Anti-ZEN Immunomagnetic Nanoparticles}

The anti-ZEN mAb was purified $(4 \mathrm{mg} / \mathrm{mL})$ and titrated with indirect ELISA. Indirect ELISA based on biotin-streptavidin-HRP system showed that anti-ZEN mAb was successfully conjugated to the magnetic nanoparticles (Figure 3): $20 \mu \mathrm{g}$ per mg magnetic nanoparticles as analyzed by BCA method on the protein concentration of the reaction solution before and after coupling.

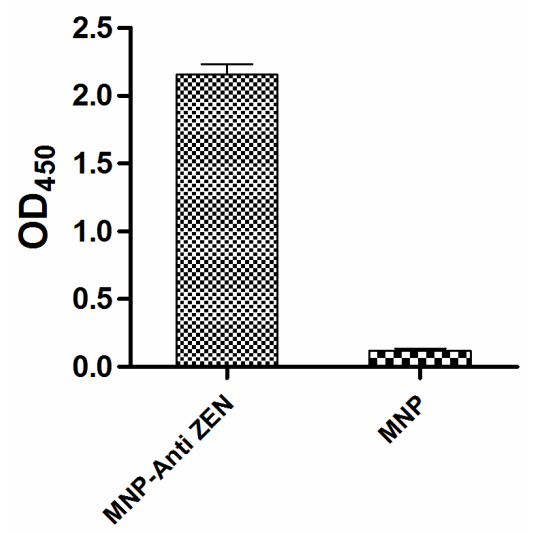

Figure 3. Identification of the immunomagnetic nanoparticles cross-linked with anti-zearalenone monoclonal antibody (MNP-anti-ZEN) by indirect MNP-bsELISA.

\subsection{Optimization of Indirect Competitive MNP-bsELISA for Quantitation of ZEN}

In MNP-bsELISA, the amounts of immunomagnetic nanoparticles and ZEN-BSA-Biotin would significantly affect the assay performance. Higher concentrations of nanoparticles could cause high background and poor sensitivity. Checkerboard titration showed that the optimum dilution of the nanoparticles was 1:100 and the optimal concentration of the ZEN-BSA-Biotin was $0.0025 \mu \mathrm{g} / \mathrm{mL}$. Strep-HRP was optimal at 1:2000 (or $0.5 \mu \mathrm{g} / \mathrm{mL}$ ), and incubation time optimal at $45 \mathrm{~min}$ (Figure 4). 


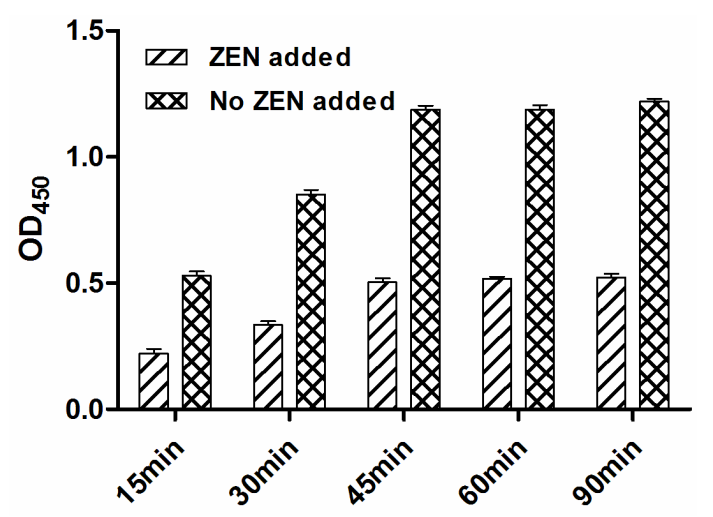

Figure 4. Determination of suitable incubation time by indirect ELISA with (+ZEN) or without $(-\mathrm{ZEN})$ addition of zearalenone.

\subsection{Specificity Study}

Using the optimized MNP-bsELISA method, the cross-reactivities with the ZEN analogues ( $\alpha$-zearalanol, zearalanone, $\alpha$-zearalenol, $\beta$-zearalenol and $\beta$-zearalanol) were $27 \%, 15 \%, 11 \%, 0.7 \%$ and $0.4 \%$, respectively. The same antibody (mAb 2C9) was found to have $16 \%$ cross-reactivity on average with other ZEN analogues in ic-ELISA (the cross-reactivities with $\alpha$-zearalanol, zearalanone and $\beta$-zearalanol were $32 \%, 17 \%$ and $0.1 \%$ ). Others also found cross-reactivity with ZEN analogues [35-37]. High structural similarity between ZEN and its analogues was the apparent reason. Such level of cross-reactivity in this method was relatively low and can be accepted. No cross-reactivity $(<0.01 \%)$ was observed with other mycotoxins (AFB1, FB1, DON and OTA) which usually occur together in cereal and feed samples. These results indicated that this novel method has good specificity.

\subsection{Calibration Curve and Matrix Interference Analysis}

A calibration curve of the MNP-bsELISA was prepared under the optimized conditions and calculated with the GraphPad5 software (GraphPad Software, La Jolla, CA, USA) (Figure 5). The linear range of detection was calculated as the concentration of ZEN leading to $20 \%-85 \%$ inhibition according to the previous study [38]. The limit of detection (LOD) was calculated as the average signal corresponding to three standard deviations from the signals of ZEN-free corn samples $(n=5)$ [39,40]. The regression equation for quantification of ZEN was $y=-0.4287 x+0.3132\left(R^{2}=0.9904\right)$. The working range was $0.7-24.1 \mu \mathrm{g} / \mathrm{kg}$. The detection limit was $0.4 \mu \mathrm{g} / \mathrm{kg}$, and $\mathrm{IC}_{50}$ (50\% inhibition of the maximum OD value) was $3.7 \mu \mathrm{g} / \mathrm{kg}$. A conventional indirect competitive ELISA (ic-ELISA) based on this antibody was also established in our laboratory [34], the regression equation was $y=-0.3909 x+0.5367\left(R^{2}=0.9940\right)$ with the working range from $0.21-9.76 \mathrm{ng} / \mathrm{mL}, \mathrm{IC}_{50}$ at $1.24 \mathrm{ng} / \mathrm{mL}$ and limit of detection at $0.12 \mathrm{ng} / \mathrm{mL}$. Table 1 shows that the MNP-bsELISA is more sensitive and faster than ic-ELISA using the same mAb 2C9 [34] and has an advantage of lower detection limit than previous reported methods such as ELISAs, fluorescence assay and array immunoassay [39-42]. Assays based on immunosensor [35] require expensive equipment, time-consuming procedures and skilled operators and are not suitable for rapid detection of toxins, although it has greater sensitivity. In the MNP-bsELISA, the signal was enhanced and the sensitivity was improved by combining using of magnetic nanoparticles and biotin-streptavidin system. With increases in binding and competition efficiency, the reaction time also decreased. 
Methanol in the extracted solution buffer and the components in the sample extracts, including proteins and vitamins, are known to affect the immuno-reagents and immune-reactions $[43,44]$. In this study, matrix interference was determined by comparing a calibration curve prepared in PBS with those obtained in serial diluted ZEN-free corn sample extracts. Figure 6 shows that all dilutions of the extracts with PBS reducing the matrix interference. Because these curves were all superimposed, we suggest that 1:1 dilution is enough to minimize the matrix effect. Matrix interference was often excluded by substantial dilution of the sample prior to analysis according to previous studies [45,46]. However, higher dilution would cause proportional reduction of the assay sensitivity. Therefore, a 1:1 dilution was selected for recovery test and detection of ZEN in cereal and feed samples in this study.

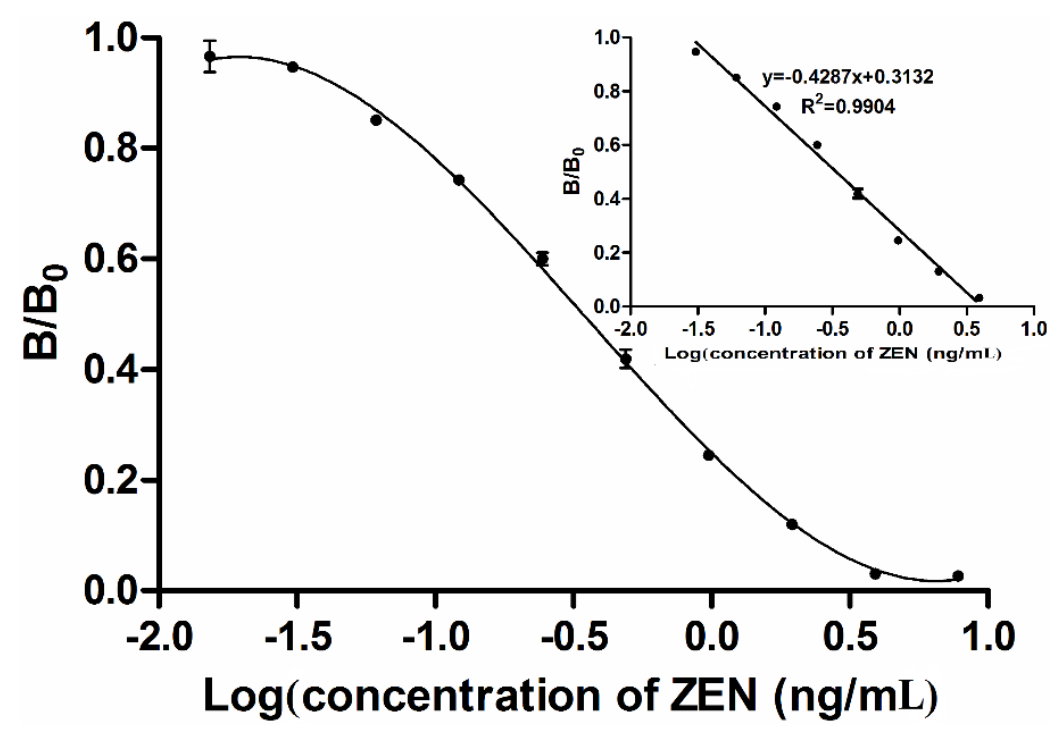

Figure 5. Tri-parametric curve fitting of log concentration of zearalenone $v s$. inhibition index by MNP-bsELISA. The insert shows the standard curve for quantification of zearalenone.

Table 1. Comparison of different analytical methods for detection of zearalenone.

\begin{tabular}{|c|c|c|c|c|c|}
\hline Methods & $\begin{array}{c}\text { Detection } \\
\text { Time }\end{array}$ & $\begin{array}{c}\text { Limit of } \\
\text { Detection }\end{array}$ & $\mathrm{IC}_{50}$ & Working Range & Ref. \\
\hline DC-ELISA & $1 \mathrm{~h}$ & $0.15 \mathrm{ng} / \mathrm{mL}$ & $1.13 \mathrm{ng} / \mathrm{mL}$ & $41.0-909.8 \mu \mathrm{g} / \mathrm{kg}$ & {$[40]$} \\
\hline ic-ELISA & $2 \mathrm{~h}$ & $0.8 \mathrm{ng} / \mathrm{mL}$ & - & $0.8-150 \mathrm{ng} / \mathrm{mL}$ & {$[41]$} \\
\hline Immunosensor assay & - & $0.007 \mathrm{ng} / \mathrm{mL}$ & - & $0.019-0.422 \mathrm{ng} / \mathrm{mL}$ & {$[35]$} \\
\hline Fluorescence assay & - & $137 \mu \mathrm{g} / \mathrm{kg}$ & - & $150-1000 \mu \mathrm{g} / \mathrm{kg}$ & [39] \\
\hline Array Immunoassay & 1.5 & $0.51 \mathrm{ng} / \mathrm{mL}$ & $2.1 \mathrm{ng} / \mathrm{mL}$ & $0.73-6.8 \mathrm{ng} / \mathrm{mL}$ & {$[42]$} \\
\hline mAb 2 C9 based ic-ELISA & $2 \mathrm{~h}$ & $0.12 \mathrm{ng} / \mathrm{mL}$ & $1.24 \mathrm{ng} / \mathrm{mL}$ & $0.21-9.76 \mathrm{ng} / \mathrm{mL}$ & {$[34]$} \\
\hline mAb 2C9 based MNP-bsELISA & 1.5 & $0.04 \mathrm{ng} / \mathrm{mL}$ & $0.37 \mathrm{ng} / \mathrm{mL}$ & $0.07-2.41 \mathrm{ng} / \mathrm{mL}$ & This study \\
\hline
\end{tabular}




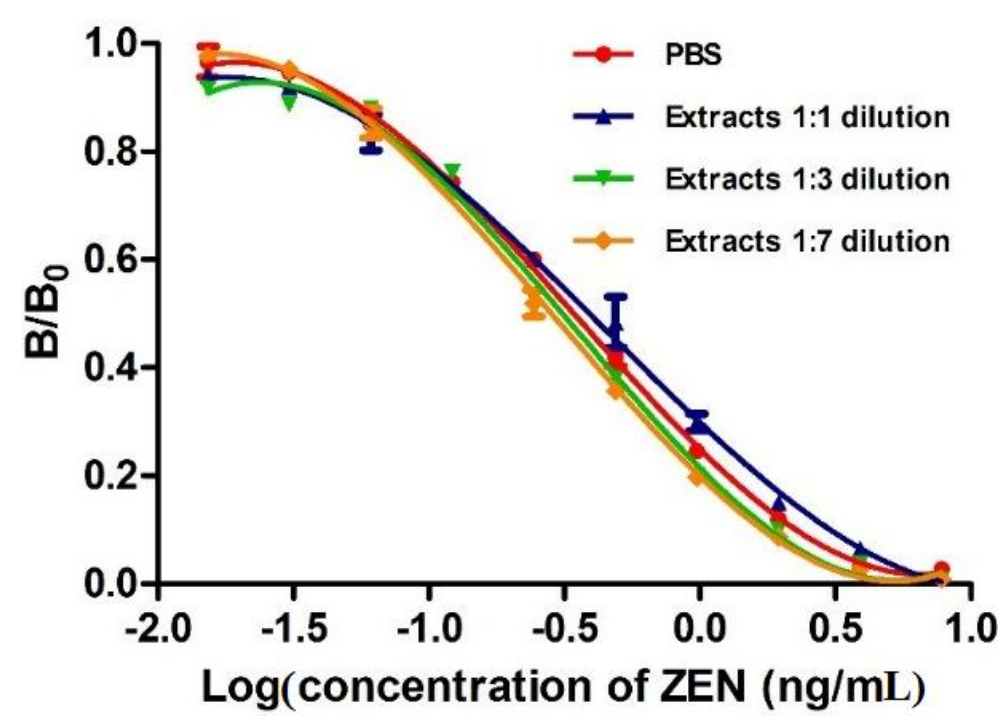

Figure 6. Analysis of matrix interferences by different dilutions, with PBS, of corn extracts spiked with different concentrations of zearalenone by MNP-bsELISA.

\subsection{Recovery Rates of ZEN in Spiked Corn Samples and Detection of ZEN in Natural Samples}

A ground corn sample without ZEN contamination was spiked with different concentrations of ZEN, and the recovery rates were determined by MNP-bsELISA. Table 2 shows that the recovery rates of intra-assay and inter-assay ranged from $92.8 \% \pm 6.9 \%$ to $111.9 \% \pm 6.7 \%$ and $91.7 \% \pm 7.3$ to $114.5 \% \pm 6.4 \%$, respectively, in spiked corn samples. The coefficients of variation (CVs) were $3.1 \%-7.5 \%$ for intraday comparisons and $4.6 \%-8.7 \%$ for interday comparisons. These results indicate that the method has good recovery from spiked samples with high precision.

Table 2. Recovery and coefficient of variances from corn samples spiked with different levels of zearalenone by MNP-bsELISA.

\begin{tabular}{cccccc}
\hline \multirow{2}{*}{ Samples } & $\begin{array}{c}\text { Spiked Level } \\
(\boldsymbol{\mu g} / \mathbf{k g})\end{array}$ & $\boldsymbol{n}$ & Measured $(\boldsymbol{\mu g} / \mathbf{k g})$ & Recovery $(\%)^{\mathbf{C V}^{\mathbf{b}}(\boldsymbol{\%})}$ \\
\cline { 3 - 6 } & 1.25 & 3 & $1.18 \pm 0.04$ & $94.5 \pm 2.9$ & 3.1 \\
2 & 2.5 & 3 & $2.32 \pm 0.18$ & $92.8 \pm 6.9$ & 7.5 \\
3 & 5 & 3 & $5.42 \pm 0.26$ & $108.4 \pm 5.2$ & 4.8 \\
4 & 10 & 3 & $11.19 \pm 0.67$ & $111.9 \pm 6.7$ & 6.1 \\
5 & 20 & 3 & $21.43 \pm 1.13$ & $107.2 \pm 5.6$ & 5.3 \\
\hline
\end{tabular}

${ }^{\mathrm{a}}$ Inter-assay variation was determined each spiked level on 3 days; ${ }^{\mathrm{b}}$ Coefficient of variation.

The natural samples were analyzed using both the MNP-bsELISA and LC-MS/MS. The results are shown in Table 3 (only positive samples are listed). Given that the detection limit of LC-MS/MS was $5 \mathrm{ug} / \mathrm{kg}$, levels of ZEN below this could not be detected, but could be detected by MNP-bsELISA. Ten samples were subjected to quantitative analysis by both methods. The relationship between MNP-bsELISA and LC-MS/MS results for ZEN in cereal and feed samples was assessed by regression analysis, MNP-bsELISA $=0.395+0.9599$ LC-MS/MS $\left(R^{2}=0.9283\right)$, indicating a good agreement between the two methods (Figure 7). These results demonstrate that the developed MNP-bsELISA can be used for ZEN detection in cereal and feed samples. 
Table 3. Comparison of MNP-bsELISA with LC-MS/MS for detection of zearalenone in cereal and feedstuff samples.

\begin{tabular}{|c|c|c|}
\hline Samples & MNP-bsELISA $(\mu \mathrm{g} / \mathrm{kg})$, Mean $\pm \mathrm{SD}^{\mathrm{a}}$ & LC-MS/MS $(\mu \mathrm{g} / \mathrm{kg})$, Mean \pm SD \\
\hline Corn 1 & $7.96 \pm 0.49$ & $9.99 \pm 0.08$ \\
\hline Corn 2 & $12.47 \pm 0.66$ & $14.01 \pm 0.45$ \\
\hline Corn 3 & $14.61 \pm 0.37$ & $14.27 \pm 0.15$ \\
\hline Corn 4 & $11.61 \pm 0.46$ & $10.91 \pm 0.28$ \\
\hline Corn 5 & $3.02 \pm 0.19$ & $-b$ \\
\hline Wheat 1 & $15.34 \pm 0.39$ & $16.67 \pm 0.05$ \\
\hline Wheat 2 & $3.76 \pm 0.21$ & $-b$ \\
\hline Wheat 3 & $12.31 \pm 0.26$ & $14.41 \pm 0.35$ \\
\hline Feedstuff 1 & $19.73 \pm 1.69$ & $21.25 \pm 0.11$ \\
\hline Feedstuff 2 & $16.19 \pm 0.71$ & $17.52 \pm 0.29$ \\
\hline Feedstuff 3 & $14.13 \pm 0.77$ & $14.84 \pm 0.28$ \\
\hline Feedstuff 4 & $18.77 \pm 1.19$ & $19.35 \pm 0.16$ \\
\hline
\end{tabular}

${ }^{\mathrm{a}} \mathrm{SD}$, standard deviation $(n=3) .{ }^{\mathrm{b}}-$, not detected.

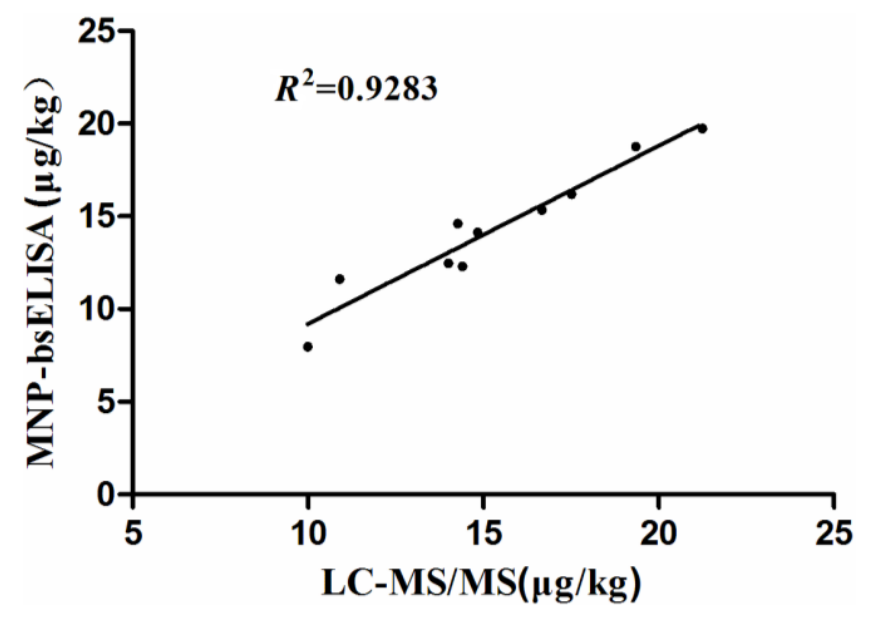

Figure 7. Correlation of results obtained by both MNP-bsELISA and LC-MS/MS for zearalenone detection in natural cereal and feed samples.

In conclusion, a novel enzyme-linked immunosorbent assay to determine ZEN levels in cereal and feed samples was developed using a system that involves magnetic nanoparticles and biotin/streptavidin-HRP (MNP-bsELISA). The method was more sensitive and rapid than conventional ELISA and had good correlation with the LC-MS/MS in quantifying zearalenone in cereal and feed samples. As a new strategy for the detection of low-molecular-weight analytes, this novel method is suitable for rapid detection of zearalenone in cereal and feed samples in relevant laboratories once it is optimized as a kit and could also be easily extended to rapid detection of other mycotoxins and biological analytes.

\section{Experimental Section}

\subsection{Chemicals and Reagents}

Zearalenone (ZEN), fumonisin B1 (FB1), aflatoxinB1 (AFB1), ochratoxin A (OTA), deoxynivalenol (DON), carboxymethoxylamine hemihydrochloride (CMO), bovine serum albumin (BSA), 
$N, N^{\prime}$-dicyclohexylcarbodiimide (DCC), $N$-hydroxy-succinimide (NHS), 2-Morpholinoethanesulfonic acid (MES) and $N, N$-Dimethylformamide (DMF) were purchased from the Sigma Chemical Co. (St. Louis, MO, USA). ZEN analogues ( $\alpha$-zearalanol, $\alpha$-zearalenol, $\beta$-zearalanol, $\beta$-zearalenol, and zearalanone) were purchased from UcallM Biotechnology Co. (Wuxi, Jiangsu, China). A hybridoma cell line secreting monoclonal antibodies against ZEN (mAb 2C9) was prepared in our laboratory. EZ-Link sulfo-NHS-LC-Biotinylation Kit (21435) was purchased from Thermo Fisher Scientific (Wyman Street, Waltham, MA, USA), Streptavidin-horseradish peroxidase (Streptavidin-HRP) was obtained from Anaspec (Fremont, CA, USA). Magnetic nanopaticles M-270 carboxylic acid (14305D) was obtained from Invitrogen (Carlsbad, CA, USA). Other reagents of analytical grade, including 3,3,5,5-tetramethylbenzidine (TMB, as ELISA substrate), were purchased from Sinopharm Chemical Reagent Co. Ltd. (Shanghai, China). Both the ZEN-free and naturally contaminated cereal and feed samples were provided by the Zhejiang Entry-Exit Inspection and Quarantine Bureau (Hangzhou, Zhejiang, China).

\subsection{Equipment}

The 96-well plates for microsphere-based ELISA were purchased from Tianlong industry (Haimen, Zhejiang, China); the $37^{\circ} \mathrm{C}$ incubator from Thermo Scientific (Waltham, MA, USA); the horizontal shaker (Vortex 4 basic) from IKA (Staufen, Germany); the magnetic separator (MS-12) from Bangs laboratories (Fishers, IN, USA); Spectra Max M2 micro-plate reader (Molecular Devices, Sunnyvale, CA, USA) was used for absorbance measurement; the ultraperformance liquid chromatography (UPLC) system and UPLC BEH C18 column were supplied by Waters (Milford, MA, USA); the QTrap MS/MS system was obtained from Applied Biosystems (Foster City, CA, USA).

\subsection{Synthesis of the ZEN-BSA Conjugate}

The ZEN-BSA conjugate was synthetized as described previously with slight modifications $[47,48]$. Zearalenone $(1.0 \mathrm{mg})$ and carboxymethoxylamine hemihydrochloride $(2.0 \mathrm{mg})$ were dissolved in $1.0 \mathrm{~mL}$ pyridine and stirred for $24 \mathrm{~h}$ at room temperature (RT). The reaction mixture was then dried under vacuum, and the residue was dissolved in $4 \mathrm{~mL}$ of distilled water ( $\mathrm{pH}$ adjusted to 8.0 with sodium hydroxide). Free zearalenone was removed from the water phase by three partitions with benzene $(3.0 \mathrm{~mL})$. The reaction mixture in the aqueous phase was then precipitated by addition of $\mathrm{HCI}(\mathrm{pH} 3.0)$ and extracted four times with $10 \mathrm{~mL}$ of ethyl acetate. The extract was dehydrated over anhydrous sodium sulfate and dried under vacuum. The residue was dissolved in $0.4 \mathrm{~mL}$ of anhydrous tetrahydrofuran and added to $6.0 \mathrm{mg}$ DCC and $3.0 \mathrm{mg}$ NHS. The mixture was allowed to react for $1 \mathrm{~h}$ by shaking gently at RT, and then centrifuged at $2500 \times g$ for $30 \mathrm{~min}$. The precipitate was discarded and the aquaous phase was dried under vacuum. The final derivative was dissolved in $2.0 \mathrm{mLDMF}$. BSA (20 mg) was dissolved in $2.0 \mathrm{~mL}$ of $130 \mathrm{mM}$ phosphate buffer ( $\mathrm{pH}$ 7.4). The activated-ZEN was added drop-wise to the BSA solution. Reaction was allowed to proceed for $1 \mathrm{~h}$ by gently shaking at RT. The solution was centrifuged to discard the precipitates. Finally, the supernatant was dialyzed against $0.01 \mathrm{~mol} / \mathrm{LPBS}(\mathrm{pH} 7.4)$ at $4{ }^{\circ} \mathrm{C}$ for $72 \mathrm{~h}$ to remove the residual free ZEN and DMF. The ZEN-BSA conjugate was confirmed by reacting with anti-ZEN mAb using indirect ELISA and Western blotting. BSA was used as control. The final product was stored at $-20^{\circ} \mathrm{C}$ for later use. 


\subsection{Biotinylation of ZEN-BSA and Identification}

Biotinylated ZEN-BSA was synthetized as described by the supplier (Thermo scientific; 21435). ZEN-BSA was dissolved in reaction buffer (0.01 M PBS, pH 7.4), and mixed with sulfo-NHS-LC-biotin by slow addition. The solution was allowed to react for $2 \mathrm{~h}$ at RT. The biotinylated derivative was purified by gel filtration on Zeba ${ }^{\mathrm{TM}}$ Spin Desalting Columns (Thermo scientific, Waltham, MA, USA; 89891). The level of biotinylation was measured by HABA (4'-hydroxyazobenzene-2-carboxylic acid) competition assay according to previous studies [49,50].

\subsection{Preparation of Immunomagnetic Nanoparticles}

The immunomagetic nanoparticles were synthetized as described by the supplier (Invitrogen, Waltham, MA, USA; 14305D). The magnetic nanoparticle stock solution $(100 \mu \mathrm{L})$ was transferred to a new Eppendorf tube which was then placed in a magnetic field to separate the nanoparticles from the storage solution. The nanoparticles were washed twice with coating buffer (25 mM MES buffer containing $0.05 \%$ Tween $20(v / v), \mathrm{pH} 5.0)$ (This buffer was prepared using the reagent mentioned in Section 3.1) before covalent coupling with the anti-ZEN antibody. EDC and NHS solutions (each at a concentration of $50 \mathrm{mg} / \mathrm{mL}$ ) were prepared in coating buffer immediately before use. A volume of $50 \mu \mathrm{L}$ each of EDC and NHS solutions were added to the washed magnetic nanoparticles and incubated with slow rotation at RT for $30 \mathrm{~min}$. The tube containing the reaction mixtures was placed in a magnetic field to remove the supernatant and washed twice with coating buffer. The purified anti-ZEN monoclonal antibody $(100 \mu \mathrm{g})$ in coating buffer $(100 \mu \mathrm{L})$ was added to the activated nanoparticles and incubated for at least 30 min at $\mathrm{RT}$, or $2 \mathrm{~h}$ at $4{ }^{\circ} \mathrm{C}$ with slow tilting rotation. The antibody-coated nanoparticles were washed with washing buffer (0.01 M PBS containing $0.02 \%$ Tween $20(v / v)$, pH 7.4) (This buffer was prepared using the reagent mentioned in Section 3.1). In order to quench the non-reacted activated carboxylic acid groups, the coated nanoparticles were incubated with quenching buffer (50 $\mathrm{mM}$ Tris containing $0.05 \%$ Tween $20[v / v], \mathrm{pH} 7.4$ ) (This buffer was prepared using the reagent mentioned in Section 3.1) for $15 \mathrm{~min}$ at RT with slow rotation. The antibody-coated magnetic nanoparticles (MNP-anti-ZEN) (Figure 1A) were then separated from the washing buffer and resuspended in storage buffer (washing buffer containing $0.5 \% \mathrm{BSA}(v / v), \mathrm{pH} 7.4$ ) (This buffer was prepared using the reagent mentioned in Section 3.1). Conjugation was confirmed by indirect ELISA using biotinylated ZEN-BSA and Streptavidin-HRP. Coating efficiency was measured by analyzing the antibody concentration before and after coupling using the BCA (bicinchoninic acid) protein assay.

\subsection{Optimization of the MNP-bsELISA}

Dilutions of MNP-Anti ZEN, concentrations of the ZEN-BSA-biotin and Strep-HRP were optimized by checkerboard titration design with an $\mathrm{OD}_{450}$ value of about 1.0 in MNP-bsELISA. Optimization conditions included dilutions of MNP-anti ZEN from 1:25, 1:50, 1:100 to 1:200; ZEN-BSA-biotin with six 2-fold dilutions from $0.01 \mu \mathrm{g} / \mathrm{mL}$; and Strep-HRP from 1:1000, 1:2000, 1:4000, 1:8000 to 1:16,000. Incubation times for the competition reaction were 15, 30, 45, 60, 75 and $90 \mathrm{~min}$. 


\subsection{Development of Indirect Competitive MNP-bsELISA}

MNP-Anti ZEN (10 $\mu \mathrm{L})$ diluted with storage buffer, $70 \mu \mathrm{L}$ ZEN-BSA-biotin solution and $70 \mu \mathrm{L}$ of ZEN standard solution at different concentrations (serial 2-fold dilution from 7.8-0.015 ng/mL, and zero control) were added to the 96-well plate, each concentration in triplicate wells. The plate was shaken at $1000 \mathrm{rpm}$ for $45 \mathrm{~min}$ at $37{ }^{\circ} \mathrm{C}$ and then placed on a magnetic base to precipitate the nanoparticles. The plate was washed three times with washing buffer, each followed by magnetic separation. Strep-HRP $(100 \mu \mathrm{L}, 0.5 \mu \mathrm{g} / \mathrm{mL})$ was added and the plate was further subjected to shaking incubation at $37{ }^{\circ} \mathrm{C}$ for $45 \mathrm{~min}$. The inmunomagnetic nanoparticle complexes were then separated on a magnetic base and washed three times. The substrate TMB $(100 \mu \mathrm{L})$ was added. Stop solution $\left(2 \mathrm{M} \mathrm{H}_{2} \mathrm{SO}_{4}\right.$, $50 \mu \mathrm{L}$ ) was pipetted after 10 min of shaking incubation at $37^{\circ} \mathrm{C}$. $\mathrm{OD}_{450}$ values were determined on the Spectra Max M2 micro-plate reader. The calibration curve for MNP-bsELISA was prepared with the GraphPad 5 software: $x$-axis represents the $\log$ concentration of $\mathrm{ZEN}(\mathrm{ng} / \mathrm{mL})$ and $y$-axis $\left(\mathrm{B} / \mathrm{B}_{0}\right)$, the division of $\mathrm{OD}_{450}$ value of standard solutions by $\mathrm{OD}_{450}$ at $0 \mathrm{ng} / \mathrm{mL}$.

\subsection{Specificity}

To evaluate the specificity of MNP-bsELISA, cross-reactivity of the anti-ZEN monoclonal antibody coated nanoparticles with five ZEN analogues and several other mycotoxins (AFB1, FB1, DON and OTA) were determined. ZEN analogues include $\alpha$-zearalanol, $\alpha$-zearalenol, $\beta$-zearalanol, $\beta$-zearalenol, and zearalanone. The cross-relativities were determined using the individual analogue, respectively. Using MNP-bsELISA, the calibration curves of ZEN with different concentrations were established first, and then different concentrations of each analyte instead of ZEN were mixed with the same volume of the ZEN-BSA-Biotin concentration $(0.0025 \mu \mathrm{g} / \mathrm{mL})$, which had been optimized in the earlier procedure. The calibration curves were prepared by GraphPad 5 software. Then the $\mathrm{IC}_{50}$ (50\% inhibition) for each analyte was calculated respectively. Cross-reactivity of the anti-ZEN monoclonal antibody coated nanoparticles with each compound was evaluated using the following formula:

$$
\text { Cross-reactivity }(\%)=\left(\mathrm{IC}_{50} \text { of } \mathrm{ZEN}\right) /\left(\mathrm{IC}_{50} \text { of other analytes }\right) \times 100 \%
$$

\subsection{Elimination of Matrix Interference and Recovery of Spiked Samples}

Matrix effect, one of the most common challenges of immunoassays of corn and feed samples analysis, was evaluated in this study by comparing several calibration curves obtained in serial diluted ZEN-free corn sample extracts with that prepared using PBS buffer alone according to previous studies $[45,46]$.

The ZEN-free corn samples were provided by the Zhejiang Entry-Exit Inspection and Quarantine Bureau after been tested by LC-MS/MS. Before spiking and recovery tests, the ZEN-free corn samples were ground and dried by overnight incubation in a $60{ }^{\circ} \mathrm{C}$ incubator. The standard ZEN solution was spiked at levels of $1.25,2.5,5,10$ and $20 \mu \mathrm{g} / \mathrm{kg}$ to ground corn samples by dropwise addition, mixed thoroughly and allowed to stand at RT overnight. Five grams of each spiked samples was placed into a $50-\mathrm{mL}$ plastic centrifuge tube. The samples were extracted with $25 \mathrm{~mL}$ of methanol/water $(7 / 3, v / v)$ at RT by vigorous vortexing for $3 \mathrm{~min}$. After centrifugation at $3500 \times g$ for $10 \mathrm{~min}$, the supernatant samples were used in MNP-bsELISA for calculation of recovery rates. To evaluate the precision of this novel method, each sample was measured three times in one day and repeated three times on different days. 


\subsection{Detection of Natural Samples by MNP-bsELISA and LC-MS/MS}

Fifty-six natural samples (including corn, wheat and feedstuff) were analyzed using MNP-bsELISA and LC-MS/MS. Each sample was tested in triplicate to calculate standard deviation. For the detection by MNP-bsELISA, the natural samples were extracted as the spiked samples and diluted 1:1 with PBS before detected. Procedures used for LC-MS/MS were as follows: before extraction, the natural samples were ground and dried overnight in a $60{ }^{\circ} \mathrm{C}$ incubator. First, ten gram of each samples was extracted with solvent mixture (40 mL, acetonitrile/water/acetic acid, 79:20:1, v/v/v) by vigorous shaken on a horizontal shaker for $60 \mathrm{~min}$ at room temperature. Second, the samples were centrifuged at $2500 \mathrm{~g}$ for $20 \mathrm{~min}$ after standing for $10 \mathrm{~min}$. The supernatants were then mixed with the same volume of mixture (acetonitrile/water/acetic acid, 20:79:1, $v / v / v$ ), and passed through a $0.22 \mu \mathrm{m}$ filter before being injected into the LC-MS/MS instrument. Quantitative LC-MS/MS results were analyzed using Analyst software (AB SCIEX, Framingham, MA, USA). The correlation between the two assays was investigated using linear regression (Microsoft Excel software, Redmond, WA, USA; 2010 version).

\section{Acknowledgments}

This work is supported by the " 863 " High-Tech program under the Chinese Ministry of Science and Technology (2012AA101602) and National Natural Science Foundation of China (31571932).

\section{Author Contributions}

Xian Zhang, Xin Wang, Mengjiao Sun, Yaxian Yan and Weihuan Fang conceived and designed the experiments; Xian Zhang, Xin Wang and Mengjiao Sun conducted the experiments and analyzed the data; Jianhe Sun and Xiaofeng Zhang contributed the materials; Xian Zhang wrote the manuscript; Xiaoliang Li, Houhui Song, and Weihuan Fang supervised the work and revised the manuscript.

\section{Conflicts of Interest}

The authors declare no conflict interest.

\section{References}

1. Kim, I.-H.; Son, H.-Y.; Cho, S.-W.; Ha, C.-S.; Kang, B.-H. Zearalenone induces male germ cell apoptosis in rats. Toxicol. Lett. 2003, 138, 185-192.

2. Placinta, C.; D'mello, J.; Macdonald, A. A review of worldwide contamination of cereal grains and animal feed with fusarium mycotoxins. Anim. Feed Sci. Technol. 1999, 78, 21-37.

3. Miller, J.D. Mycotoxins in grain. Rev. Ins. Med. Trop. Sao Paulo 1994, 36, 326-326.

4. Rodricks, J.V.; Hesseltine, C.W.; Mehlman, M.A. Mycotoxins in Human and Animal Health. In Proceedings of a Conference Convened at University of Maryland, College Park, MD, USA, 4-8 October 1976.

5. Collins, T.F.; Sprando, R.L.; Black, T.N.; Olejnik, N.; Eppley, R.M.; Alam, H.Z.; Rorie, J.; Ruggles, D.I. Effects of zearalenone on in utero development in rats. Food Chem. Toxicol. 2006, 44, 1455-1465. 
6. Panel, E.C. Scientific opinion on the risks for public health related to the presence of zearalenone in food. EFSA J. 2011, 9, 2197.

7. EFSA, J. Opinion of the scientific panel on contaminants in the food chain on a request from the commission related to zearalenone as undesirable substance in animal feed. EFSA J. 2004, 89, 1-35.

8. Šegvić Klarić, M.; Cvetnić, Z.; Pepeljnjak, S.; Kosalec, I. Co-occurrence of aflatoxins, ochratoxin A, fumonisins, and zearalenone in cereals and feed, determined by competitive direct enzyme-linked immunosorbent assay and thin-layer chromatography. Arh. Higijenu Rada Toksikol. 2009, 60, 427-433.

9. Tsiplakou, E.; Anagnostopoulos, C.; Liapis, K.; Haroutounian, S.A.; Zervas, G. Determination of mycotoxins in feedstuffs and ruminant's milk using an easy and simple LC-MS/MS multiresidue method. Talanta 2014, 130, 8-19.

10. Zhang, K.; Wong, J.W.; Krynitsky, A.J.; Trucksess, M.W. Determining mycotoxins in baby foods and animal feeds using stable isotope dilution and liquid chromatography tandem mass spectrometry. J. Agric. Food Chem. 2014, 62, 8935-8943.

11. Lacina, O.; Zachariasova, M.; Urbanova, J.; Vaclavikova, M.; Cajka, T.; Hajslova, J. Critical assessment of extraction methods for the simultaneous determination of pesticide residues and mycotoxins in fruits, cereals, spices and oil seeds employing ultra-high performance liquid chromatography-tandem mass spectrometry. J. Chromatogr. A 2012, 1262, 8-18.

12. Giovannoli, C.; Passini, C.; Di Nardo, F.; Anfossi, L.; Baggiani, C. Determination of ochratoxin a in italian red wines by molecularly imprinted solid phase extraction and HPLC analysis. J. Agric. Food Chem. 2014, 62, 5220-5225.

13. Stanker, L.H.; Scotcher, M.C.; Cheng, L.; Ching, K.; McGarvey, J.; Hodge, D.; Hnasko, R. A monoclonal antibody based capture ELISA for botulinum neurotoxin serotype B: Toxin detection in food. Toxins 2013, 5, 2212-2226.

14. Ling, S.; Pang, J.; Yu, J.; Wang, R.; Liu, L.; Ma, Y.; Zhang, Y.; Jin, N.; Wang, S. Preparation and identification of monoclonal antibody against fumonisin $\mathrm{B} 1$ and development of detection by Ic-ELISA. Toxicon 2014, 80, 64-72.

15. Li, Y.; Luo, X.; Yang, S.; Cao, X.; Wang, Z.; Shi, W.; Zhang, S. High specific monoclonal antibody production and development of an ELISA method for monitoring T-2 toxin in rice. J. Agric. Food Chem. 2014, 62, 1492-1497.

16. Shim, W.B.; Dzantiev, B.B.; Eremin, S.A.; Chung, D.H. One-step simultaneous immunochromatographic strip test for multianalysis of ochratoxin A and zearalenone. J. Microbiol. Biotechnol. 2009, 19, 83-92.

17. Kolosova, A.Y.; de Saeger, S.; Sibanda, L.; Verheijen, R.; van Peteghem, C. Development of a colloidal gold-based lateral-flow immunoassay for the rapid simultaneous detection of zearalenone and deoxynivalenol. Anal. Bioanal. Chem. 2007, 389, 2103-2107.

18. Wang, Y.-K.; Yan, Y.-X.; Ji, W.-H.; Wang, H.-A.; Li, S.-Q.; Zou, Q.; Sun, J.-H. Rapid simultaneous quantification of zearalenone and fumonisin B1 in corn and wheat by lateral flow dual immunoassay. J. Agric. Food Chem. 2013, 61, 5031-5036.

19. Alarcón, S.H.; Palleschi, G.; Compagnone, D.; Pascale, M.; Visconti, A.; Barna-Vetró, I. Monoclonal antibody based electrochemical immunosensor for the determination of ochratoxin A in wheat. Talanta 2006, 69, 1031-1037. 
20. Zhou, J.; Du, L.; Zou, L.; Zou, Y.; Hu, N.; Wang, P. An ultrasensitive electrochemical immunosensor for carcinoembryonic antigen detection based on staphylococcal protein $\mathrm{A}-\mathrm{Au}$ nanoparticle modified gold electrode. Sens. Actuators B: Chem. 2014, 197, 220-227.

21. Matsukuma, E.; Kato, Z.; Omoya, K.; Hashimoto, K.; Li, A.; Yamamoto, Y.; Ohnishi, H.; Hiranuma, H.; Komine, H.; Kondo, N. Development of fluorescence-linked immunosorbent assay for high throughput screening of interferon- $\gamma$. Allergology Int. 2006, 55, 49-54.

22. Zhang, Z.; Li, Y.; Li, P.; Zhang, Q.; Zhang, W.; Hu, X.; Ding, X. Monoclonal antibody-quantum dots CdTe conjugate-based fluoroimmunoassay for the determination of aflatoxin B1 in peanuts. Food Chem. 2014, 146, 314-319.

23. Wang, Z.; Li, H.; Li, C.; Yu, Q.; Shen, J.; De Saeger, S. Development and application of a quantitative fluorescence-based immunochromatographic assay for fumonisin B1 in maize. J. Agric. Food Chem. 2014, 62, 6294-6298.

24. Kuo, H.-T.; Yeh, J.Z.; Jiang, C.-M.; Wu, M.-C. Magnetic particle-linked anti hCG $\beta$ antibody for immunoassay of human chorionic gonadotropin (hCG), potential application to early pregnancy diagnosis. J. Immunol. Methods 2012, 381, 32-40.

25. Pappert, G.; Rieger, M.; Niessner, R.; Seidel, M. Immunomagnetic nanoparticle-based sandwich chemiluminescence-ELISA for the enrichment and quantification of E. coli. Microchim. Acta 2010, $168,1-8$.

26. Urusov, A.E.; Petrakova, A.V.; Vozniak, M.V.; Zherdev, A.V.; Dzantiev, B.B. Rapid immunoenzyme assay of aflatoxin B1 using magnetic nanoparticles. Sensors 2014, 14, 21843-21857.

27. Ohne, K.; Kani, S.; Ohashi, M.; Shinkai, N.; Inoue, T.; Wakimoto, Y.; Tanaka, Y. clinical evaluation of a newly developed high-sensitive detection of hepatitis B virus surface antigen by a semi-automated immune complex transfer chemiluminescent enzyme immunoassay. Rinsho byori. Jpn. J. Clin. Pathol. 2013, 61, 787-794.

28. Smith, J.E.; Sapsford, K.E.; Tan, W.; Ligler, F.S. Optimization of antibody-conjugated magnetic nanoparticles for target preconcentration and immunoassays. Anal. Biochem. 2011, 410, 124-132.

29. Diler, E.; Obst, U.; Schmitz, K.; Schwartz, T. A lysozyme and magnetic bead based method of separating intact bacteria. Anal. Bioanal. Chem. 2011, 401, 253-265.

30. Hu, Y.; Shen, G.; Zhu, H.; Jiang, G. A class-specific enzyme-linked immunosorbent assay based on magnetic particles for multiresidue organophosphorus pesticides. J. Agric. Food Chem. 2010, 58, 2801-2806.

31. Bu, D.; Zhuang, H.S.; Yang, G.X. A sensitive enzyme-linked immunosorbent assay amplified by biotin-streptavidin system for detecting non-steroidal anti-inflammatory drug ketoprofen. J. Environ. Sci. Health Part B 2014, 49, 408-415.

32. Lin, Z.; Wang, X.; Li, Z.-J.; Ren, S.-Q.; Chen, G.-N.; Ying, X.-T.; Lin, J.-M. Development of a sensitive, rapid, biotin-streptavidin based chemiluminescent enzyme immunoassay for human thyroid stimulating hormone. Talanta 2008, 75, 965-972.

33. Zhu, Q.; Chai, Y.; Zhuo, Y.; Yuan, R. Ultrasensitive simultaneous detection of four biomarkers based on hybridization chain reaction and biotin-streptavidin signal amplification strategy. Biosens. Bioelectron. 2015, 68, 42-48. 
34. Wang, Y.; Wang, J.; Wang, Y.; Chen, Z.; Yan, Y.; Hao, Q.; Li, S.; Yu, C.; Yang, C.; Sun, J. Preparation of anti-zearalenone monoclonal antibodies and development of an indirect competitive elisa for zearalenone. Microbiol. China 2011, 38, 1793-1800.

35. Urraca, J.L.; Benito-Peña, E.; Pérez-Conde, C.; Moreno-Bondi, M.C.; Pestka, J.J. Analysis of zearalenone in cereal and swine feed samples using an automated flow-through immunosensor. J. Agric. Food Chem. 2005, 53, 3338-3344.

36. Burmistrova, N.A.; Goryacheva, I.Y.; Basova, E.Y.; Franki, A.-S.; Elewaut, D.; Van Beneden, K.; Deforce, D.; van Peteghem, C.; de Saeger, S. Application of a new anti-zearalenone monoclonal antibody in different immunoassay formats. Anal. Bioanal. Chem. 2009, 395, 1301-1307.

37. Burkin, A.; Kononenko, G.; Soboleva, N. Group-specific antibodies against zearalenone and its metabolites and synthetic analogs. Appl. Biochem. Microbiol. 2002, 38, 169-176.

38. Kido, K.; Edakuni, K.; Morinaga, O.; Tanaka, H.; Shoyama, Y. An enzyme-linked immunosorbent assay for aconitine-type alkaloids using an anti-aconitine monoclonal antibody. Anal. Chim. Acta 2008, 616, 109-114.

39. Chun, H.S.; Choi, E.H.; Chang, H.-J.; Choi, S.-W.; Eremin, S.A. A fluorescence polarization immunoassay for the detection of zearalenone in corn. Anal. Chim. Acta 2009, 639, 83-89.

40. Thongrussamee, T.; Kuzmina, N.; Shim, W.-B.; Jiratpong, T.; Eremin, S.; Intrasook, J.; Chung, D.-H. Monoclonal-based enzyme-linked immunosorbent assay for the detection of zearalenone in cereals. Food Addit. Contam. 2008, 25, 997-1006.

41. Zhi-hong, M. Detection on zearalenone in grains by biotin-streptavidin enzyme-linked immunosorbent assay. J. Anhui Agric. Sci. 2009, 15, 016.

42. Wang, Y.-K.; Yan, Y.-X.; Li, S.-Q.; Wang, H.-A.; Ji, W.-H.; Sun, J.-H. Simultaneous quantitative determination of multiple mycotoxins in cereal and feedstuff samples by a suspension array immunoassay. J. Agric. Food Chem. 2013, 61, 10948-10953.

43. Wang, S.; Quan, Y.; Lee, N.; Kennedy, I.R. Rapid determination of fumonisin B1 in food samples by enzyme-linked immunosorbent assay and colloidal gold immunoassay. J. Agric. Food Chem. 2006, 54, 2491-2495.

44. Wang, Y.-K.; Shi, Y.-B.; Zou, Q.; Sun, J.-H.; Chen, Z.-F.; Wang, H.-A.; Li, S.-Q.; Yan, Y.-X. Development of a rapid and simultaneous immunochromatographic assay for the determination of zearalenone and fumonisin b1 in corn, wheat and feedstuff samples. Food Control. 2013, 31, 180-188.

45. Ono, E.Y.; Kawamura, O.; Ono, M.A.; Ueno, Y.; Hirooka, E.Y. A comparative study of indirect competitive ELISA and HPLC for fumonisin detection in corn of the state of paraná, brazil. Food Agric. Immunol. 2000, 12, 5-14.

46. Quan, Y.; Zhang, Y.; Wang, S.; Lee, N.; Kennedy, I.R. A rapid and sensitive chemiluminescence enzyme-linked immunosorbent assay for the determination of fumonisin B1 in food samples. Anal. Chim. Acta 2006, 580, 1-8.

47. Gendloff, E.; Casale, W.; Ram, B.; Tai, J.; Pestka, J.; Hart, L. Hapten-protein conjugates prepared by the mixed anhydride method: Cross-reactive antibodies in heterologous antisera. J. Immunol. Methods 1986, 92, 15-20.

48. Thouvenot, D.; Morfin, R.F. Radioimmunoassay for zearalenone and zearalanol in human serum: Production, properties, and use of porcine antibodies. Appl. Environ. Microbiol. 1983, 45, 16-23. 
49. Yellepeddi, V.K.; Kumar, A.; Palakurthi, S. Biotinylated poly (amido) amine (pamam) dendrimers as carriers for drug delivery to ovarian cancer cells in vitro. Anticancer Res. 2009, 29, 2933-2943.

50. Landar, A.; Oh, J.-Y.; Giles, N.M.; Isom, A.; Kirk, M.; Barnes, S.; Darley-Usmar, V.M. A sensitive method for the quantitative measurement of protein thiol modification in response to oxidative stress. Free Radic. Biol. Med. 2006, 40, 459-468.

(C) 2015 by the authors; licensee MDPI, Basel, Switzerland. This article is an open access article distributed under the terms and conditions of the Creative Commons Attribution license (http://creativecommons.org/licenses/by/4.0/). 